\section{$\underset{\substack{\text { hommes } \\ \text { \& migrations }}}{ }$}

\section{Hommes \& migrations}

Revue française de référence sur les dynamiques

migratoires

$1297 \mid 2012$

Migrations en création

\title{
Là où vont nos pères de Shaun Tan
}

Enquêtes sur (en quête d') un pays sans nom dans l'univers du neuvième art

\section{Vincent Marie}

\section{(2) OpenEdition \\ Journals}

Édition électronique

URL : http://journals.openedition.org/hommesmigrations/1549

DOI : 10.4000/hommesmigrations.1549

ISSN : 2262-3353

Éditeur

Musée national de l'histoire de l'immigration

Édition imprimée

Date de publication : 1 mai 2012

Pagination : 78-86

ISSN : 1142-852X

Référence électronique

Vincent Marie, «Là où vont nos pères de Shaun Tan », Hommes \& migrations [En ligne], 1297 | 2012,

mis en ligne le 31 décembre 2014, consulté le 02 mai 2019. URL : http://journals.openedition.org/ hommesmigrations/1549; DOI : 10.4000/hommesmigrations.1549 


\section{Là où vont nos pères de Shaun Tan \\ Enquêtes sur (en quête $d^{\prime}$ ) un pays sans nom dans l'univers du neuvième art}

Par Vincent Marie, docteur en histoire contemporaine, chercheur associé au CHCS, université Versailles Saint-Quentin-en-Yvelines (Groupe de recherche : pour une histoire culturelle de la bande dessinée)

Dans la représentation du parcours migratoire, la bande dessinée oscille entre le réalisme et la métaphore. Avec l'Australien Shaun Tan, elle se passe même de mots pour décrire la rencontre entre un migrant de papier et un pays imaginaire. Le dessin seul, au service d'un artiste en quête d'appartenance, plonge dans l'intimité du regard du migrant. Dans son univers surréaliste murmurent les voix de ceux et celles qui font des migrations un itinéraire universel. 
(f)

Sous une apparente surface, tout projet artistique cache un vaste iceberg maintenant l'ensemble de la structure à flot...

Shaun Tan.

L'immigration est un thème qui entretient des relations singulières avec le neuvième art. Sur un temps long et à travers un large corpus, leur association permet de mettre en évidence la question de la représentation d'un sujet sensible, de ses invariances et de ses stéréotypes, mais aussi de ses évolutions voire ruptures. En effet, entre lieux communs et originalités, la bande dessinée a construit depuis près d'un siècle une "mythologie iconographique" des migrations, autrement dit un imaginaire de l'immigration qui s'appuie à la fois sur une Histoire à dimension universelle et sur des récits singuliers (autobiographique, de fiction, de reportage, de science-fiction...). Cependant, faire l'Histoire des représentations artistiques et culturelles des migrations ne doit pas seulement se borner à recenser les projections médiatiques d'une société à la vision européano-centrée (c'est-à-dire voir le sujet exclusivement à travers la nature économique des migrations ou du seul prisme franco-maghrébin...), mais s'appliquer à faire émerger des itinéraires d'artistes de différentes origines pour faire dialoguer les expériences graphiques et les témoignages humains. L'étude de la bande dessinée Là où vont nos pères et le parcours de l'Australien Shaun Tan sont ici particulièrement significatifs des liens que peuvent tisser immigration et création artistique. Dans un grand tableau intitulé The Hundred Year Picnic (Le Pique-nique de cent ans) et réalisé en 2003 pour la bibliothèque publique d'Australie-Occidentale, Shaun Tan s'inspire d'une photographie d'archives locales pour immortaliser un pique-nique familial un siècle avant l'urbanisation de la région.

Il s'agit d'une interprétation stylisée et onirique d'une famille en terre étrangère, confrontée à un paysage qui ne correspond pas avec l'héritage européen, ce dont témoignent leurs vêtements, leurs objets et leur langage corporel. Sur la toile, ces deux mondes ne semblent pas coïncider, mais pour Shaun Tan, ils ne sont pas pour autant incompatibles: "Malgré les accidents de l'Histoire, les individus ont souvent la possibilité de surmonter ces différences. Les liens créés avec un nouvel endroit peuvent être de nature profonde sans pour autant être clairement articulés, un peu comme une composition nébuleuse de couleurs, de formes et de texture $e^{(1)}$." L'artiste né en 1974 dans la banlieue nord de Pearth en Australie explique, dans cette perspective, que "de façon consciente ou non, il a toujours été attiré par les histoires de personnages qui se retrouvent perdus, déracinés dans un monde inconnu ou bien en mal d'appartenance ${ }^{(2)}$."

Ce tableau annonce déjà son prochain chef-d'œuvre: un récit muet intitulé The Arrival racontant par le dessin l'histoire d'un homme qui laisse sa femme et sa fille dans une ville que l'on devine touchée par une crise. Primé à Angoulême en 2007, le récit de ce 
père de famille parti tenter sa chance dans un pays de papier inconnu de l'autre côté de l'océan pourrait être qualifié d' "objet culturel non identifié(3)" d'autant plus qu'à l'instar du tableau réalisé pour la bibliothèque publique australienne, cet épisode pose la question centrale de l'appartenance, récurrente dans l'ceuvre de Shaun Tan. Si cette épopée graphique peut être perçue comme une aventure singulière (celle d'un homme quittant sa famille), elle est surtout révélatrice des caractéristiques universelles de tout parcours de migrant. En effet, dans le titre de l'album se lisent déjà trois thèmes essentiels de la migration : là (le pays inconnu); où vont (la migration et le sentiment d'appartenance); nos pères (le lien familial). Enquêter sur un pays sans nom, c'est aussi partir en quête car "sous une apparente surface, tout projet artistique cache souvent un iceberg maintenant la structure à flot $t^{4}$." C'est la face cachée de l'iceberg de Là où vont nos pères que nous tenterons donc de mettre à jour dans les lignes qui vont suivre....

\section{Une approche formelle et esthétique originale}

Avec Là où vont nos pères, Shaun Tan relève le défi de raconter une histoire de migration sans mots. Dans cette bande dessinée, il n'y a pas de parole, pas de phylactère, pas de texte narratif, seulement des images dont l'échelle des plans et les agencements définissent le rythme du récit. La posture de l'artiste s'articule donc sur l'un des fondements de la bande dessinée : celui de construire un récit dont la narration est avant tout visuelle oscillant entre actions rapides (par l'usage de vignettes petites et de plans rapprochés, voire de gros plans ou très gros plans) et pauses contemplatives dans le récit (par des images de paysage de différentes natures en vue panoramique ou vue d'ensemble). S'appuyant sur les pouvoirs d'une chronique silencieuse, l'artiste australien raconte l'histoire d'une migration qui trouve racine dans son imaginaire et dans son histoire personnelle. Ainsi, concernant la genèse de son projet, il explique qu' 'on me demande souvent d'où m'est venue l'idée de ce livre et, s'il n'est pas simple de répondre à cette question, je peux néanmoins affirmer que tout a commencé par de vagues images mentales d'un étranger portant une valise, bien qu'au début je n'aie pas été capable d'en tirer quoi que ce soit de significatif. Bien plus tard jai pris conscience du lien avec une thématique évidente, la migration, mais cela n'a fait qu'exacerber les problèmes qui se posaient à moi en termes de représentation artistique ${ }^{(5)}$.

Les interrogations sur le déracinement, l'identité et l'appartenance qui fondent les enjeux esthétiques de son exploration artistique s'inscrivent dans son parcours personnel. C'est en effet dans l'histoire familiale de l'auteur que l'on peut d'abord rechercher les stigmates qui imprègnent sa recherche graphique. De mère australienne (de troisième génération) aux racines irlandaises et anglaises et de père chi- 
nois (né en Malaisie de parents immigrés), Shaun Tan apparaît comme un artiste freelance déraciné et en quête d'appartenances. Si au début de sa carrière, il réalise de nombreux dessins et illustrations pour des livres de littérature jeunesse ${ }^{(6)}$, c'est surtout en 2008, avec le Fauve d'or, prix de la meilleure bande dessinée obtenu lors du festival d'Angoulême pour The Arrival ${ }^{7}$ que sa virtuosité graphique dépasse les frontières de l'Océanie. Dessinée au crayon dans les teintes sépia, Là où vont nos pères est une aventure humaine et graphique qui révèle un voyage passé. En ce sens, la bande dessinée se feuillette comme un album de photographies ; une série d'images dans un ordre chronologique illustrant la vie, le parcours de quelqu'un. Pour autant les décors pourraient être ceux de demain. Ils sont en dehors du temps. Un temps où le silence, la violence et l'inconnu s'expriment dans le

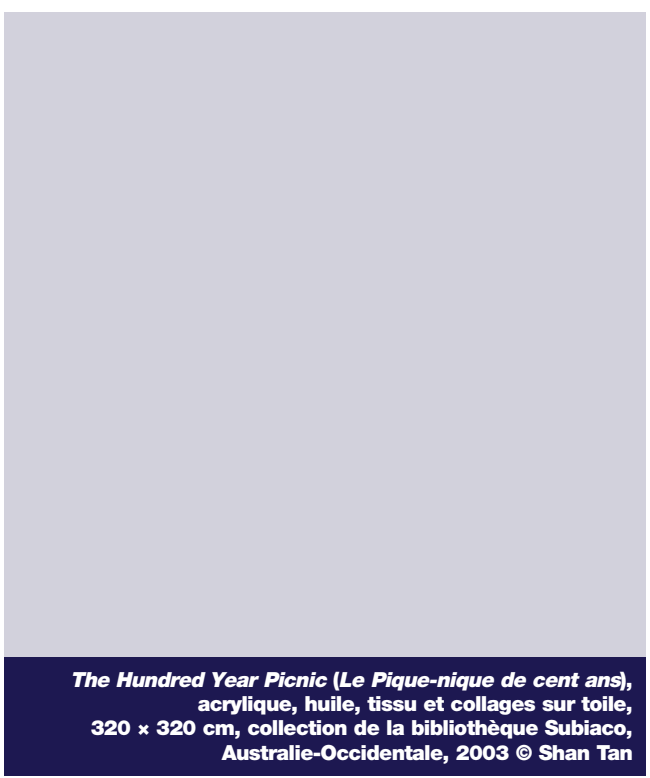
trait. L'auteur accommode objets du quotidien (un chapeau, une marmite, une horloge...) et personnages réalistes dans un monde totalement créé. Il élabore pour les lecteurs un endroit non familier, quel que soit leur âge et leur pays de résidence dans le but de mieux faire comprendre ce que signifie vivre et se déplacer dans un pays inconnu. Dans cette perspective, Shaun Tan s'est documenté "en lisant des biographies, en écoutant des entretiens enregistrés et en en conduisant un certain nombre (notamment auprès de son père), jai compris que les problèmes rencontrés par de nombreux migrants étaient parallèles à mes préoccupations de plasticien. Il y a cette lutte pour créer du sens malgré l'absence de langage écrit ou parlé, cette tendance à disséquer son sentiment de déracinement, à tout observer avec force attention et imagination ${ }^{(8)}$ '. Sa fascination pour les histoires illustrées à caractères surréalistes s'articule donc dans ce récit autour de la recherche d'une certaine forme de simplification ou de stylisation de ses figures humaines de papier. Là où vont nos pères s'inscrit de fait dans la filiation des travaux de l'artiste britannique Raymond Briggs, et du réalisme décalé pratiqué par des dessinateurs comme Chris Ware, David B. ou encore Art Spiegelman. Pour l'artiste australien, il s'agit d'atteindre une certaine forme d'universalité graphique au service d'une désorientation culturelle. 


\section{Un parcours singulier, un itinéraire universel}

Là où vont nos pères raconte une histoire de migration singulière, une aventure particulière mais dont la portée et les caractéristiques sont universelles. Découpée en six chapitres bien distincts, la bande dessinée présente les différentes étapes d'une migration atemporelle : le départ, le voyage, l'arrivée dans un pays inconnu, l'intégration... Pour Shaun Tan migrer, c'est devenir étranger dans un pays étrange. Cette réflexion trouve, sur la planche, un espace d'expression particulièrement fécond, d'autant plus qu'elle s'inscrit dans une démarche universaliste. Sous sa plume, le migrant de papier cherche des points de repère dans un monde où les plus petits détails de la vie ordinaire sont perturbants, sans parler des difficultés à saisir le sens des mots prononcés dans une langue étrangère. Dans cette optique, la profondeur artistique des images produites ici s'inscrit dans l'Histoire mondiale des migrations avec un grand $\mathrm{H}$. En effet, certaines vignettes sont plus ou moins directement inspirées des photographies provenant des archives du musée d'Ellis Island à New York comme celle de la salle d'enregistrement ou "Great Hall" d'Ellis Island prise entre 1907 et 1912. Elles forment alors de véritables icônes mémorielles. Pour Shaun Tan, le Great Hall d'Ellis Island qui a vu transiter plus de douze millions de personnes pendant sa période d'activité entre 1892 et 1954 constitue un point de repère central véritable "lieu de mémoire" iconographique au service d'un imaginaire collectif des migrations. Ainsi, sur la réinterprétation graphique de cette photographie, Shaun Tan tente 'd'amplifier la 'poésie' subtile de l'image d'origine : cette masse humaine sombre et dense, la ligne de fuite que tracent les bancs vers le drapeau central (étrange symbole d'autorité et de liberté) et l'étreinte protectrice de la voûte aux allures de cathédrale. La surexposition des fenêtres à l'étage supérieur laisse imaginer juste au-delà des portes une terre de lumière et de possibilités. Dans mon dessin, des tours au loin relâchent les immigrants à bord de ballons, tandis que le reste d'entre eux attend encore dans la pénombre du hall, en deçà de la ligne d'horizon. Cette image suggère à la fois un espèce d'accablement et un respect mêlé de crainte, le tout baignant dans l'obscurité : nous ne comprenons pas cet endroit et, seulement capables de l'interpréter à travers une grille intuitive ou affective, nous devenons aussi 'migrants' ${ }^{\text {(10)" }}$.

Par ailleurs, les photographies historiques utilisées par Shaun Tan sont aussi décontextualisées de leur régime d'historicité même si elles n'en constituent pas néanmoins un point d'ancrage fort à l'échelle du récit iconographique. L'exemple célèbre de Ned Parfett, un garçon vendeur de journaux annonçant le naufrage du Titanic est ici significatif. Shaun Tan s'inspire de cette image mais la recadre et gomme de la photographie originelle tous les indices historiques (c'est-à-dire l'annonce à la Une du journal du désastre maritime du Titanic) en remplaçant la typographie du journal par une graphie originale traduction d'une langue fictive atemporelle et volontairement incompréhensible. 
Salle d'enregistrement ou "Great Hall" d'Ellis Island, entre 1907 et 1912

๑) Ellis Island

Par ailleurs, pour le dessinateur australien maintenir le lien familial est un gage de survie. En effet, si le migrant part généralement seul, laissant derrière lui sa famille, ses proches; sa principale motivation, vient de la séparation et de l'espoir de créer un nouvel environne-

Ce même hall réinventé dans le pays imaginaire de Là où vont nos pères, Dargaud, 2008 ๑ Dargaud ment pour réunir la famille. Alors cela

lui donne la force de surmonter les obstacles rencontrés dans le pays d'accueil. Dans la bande dessinée, la photographie de famille est un objet central. Dans le contexte de la décomposition familiale liée à la migration, elle ne quitte jamais les affaires du père et exerce une fonction que l'on pourrait qualifier de "religieuse" au sens étymologique du terme ${ }^{(11)}$ et à double titre. Pour le lecteur, elle fait le lien entre les différentes parties de l'album et pour le père migrant, elle conserve de façon plus ou moins tangible le lien avec sa famille: sa femme et sa fille. Enfin, on pourrait reprocher à Shaun Tan de proposer un parcours exclusivement masculin, traduction selon Nancy Green du poids de l'historiographie de l'histoire des migrations ${ }^{(12)}$, mais c'est sans compter sur la dernière image de l'album. Cette vignette réalise en effet le tour de force d'occuper non seulement une page entière mais permet surtout d'appréhender l'histoire des migrations comme celle d'un itinéraire universel et métaphorique. La petite fille du père de famille dont on a suivi le parcours et que l'on reconnaît grâce au bonnet qu'elle porte sur la tête indique le chemin à une jeune femme migrante qui nous ait inconnue. Par le geste que sa main dessine dans la vignette elle désigne un hors-champ à la fois spatial et atemporel. 


\section{Nourrir l'imaginaire : une œuvre hors du temps?}

Le langage utilisé et l'univers onirique proposé par Shaun Tan dépassent largement les frontières. Le désarroi face à la découverte d'un autre univers, d'une autre culture et les obstacles à l'intégration sont amplifiés par le choix d'un monde imaginaire, mélange de féerie et de surréalisme. Entièrement muette, cette approche graphique permet toutefois de donner la parole à tous ces exilés qui se retrouvent perdus en terre étrangère ainsi qu'à tous ceux qui les y accueillent: "J'ai toujours été intéressé par l'idée d'une illustration indirecte ; autrement dit, il s'agit de trouver un équivalent métaphorique à un thème ou une histoire, une représentation imaginaire plutôt que littérale ${ }^{(13),}$, explique Shaun Tan. Le choix de proposer en pages de garde une série de portraits au format de photos d'identité, pour la plupart inspirés de photographies provenant des archives du musée d'Ellis Island à New

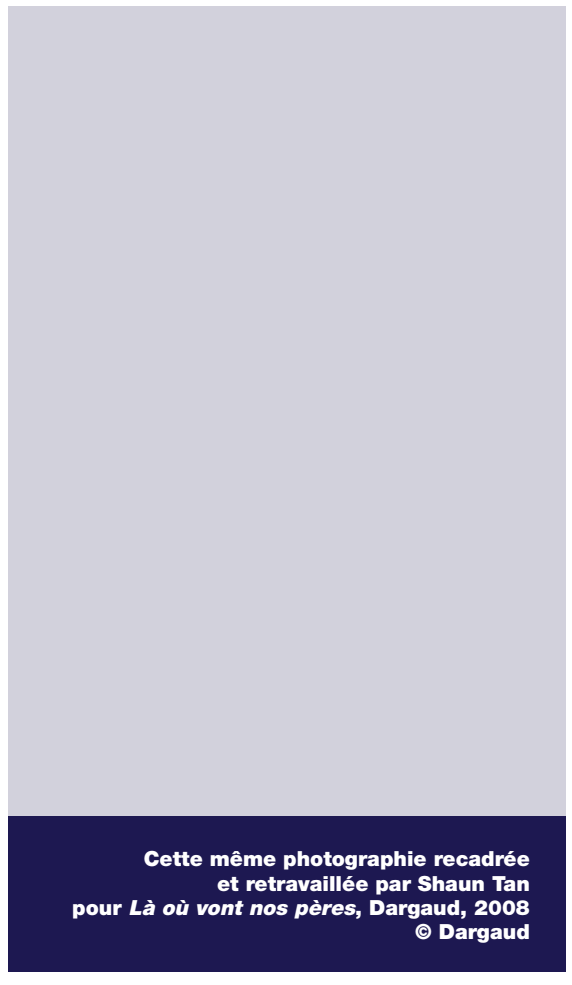

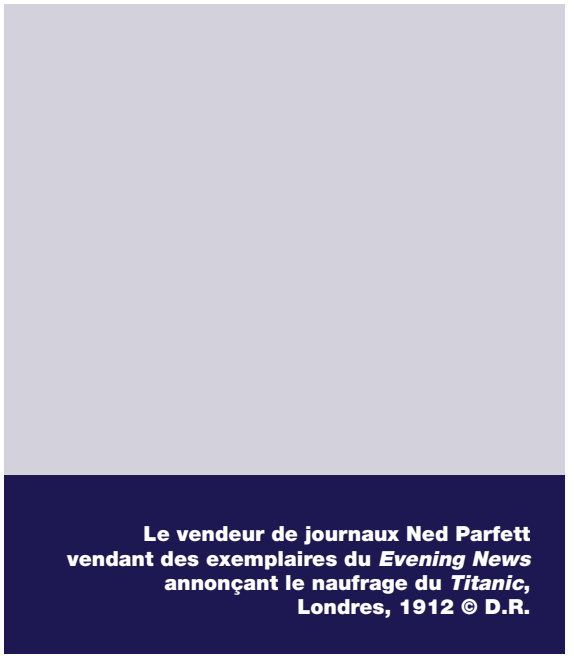

York témoigne de la volonté de redonner la parole par le dessin à ces milliers d'hommes et de femmes qui ont parfois tout quitté. Pour autant, on peut penser que Shaun Tan demeure parfois prisonnier de ses sources d'inspiration et de certaines représentations culturelles, notamment lorsqu'il met en scène le voyage du père par bateau ${ }^{(14)}$. En effet, si les mouvements de population contemporains ont été pour beaucoup une 
histoire d'eau, la traversée que l'artiste expose ici n'est-elle pas celle de l'Atlantique en relation avec les images d'Ellis Island qu'il utilise ? Image forte des migrations de masse du XIX ${ }^{\mathrm{e}}$ siècle, la traversée de l'océan est popularisée dans tous les arts : on pense aux gravures ou aux films classiques comme ceux de Chaplin (L'Émigrant) ou de Kazan (America! America !). Sous la plume de l'artiste australien et à l'aide de procédés empruntés au cinéma (notamment le travelling), la fluidité du voyage devient métaphore animée et les arrivées successives de populations sont assimilées aux vagues, aux flux ou aux courants qui les ont portées vers les rivages.

En fait, c'est surtout la capacité de Shaun Tan à jouer avec les imaginaires de ses lecteurs, à travers l'espace inter iconique des vignettes qui transcende largement l'cuvre et dessine les contours d'un "art invisible $^{(15)}$ ", structure essentielle dans la bande dessinée. La séquence d'ouverture de l'album trouve en effet ici toute sa

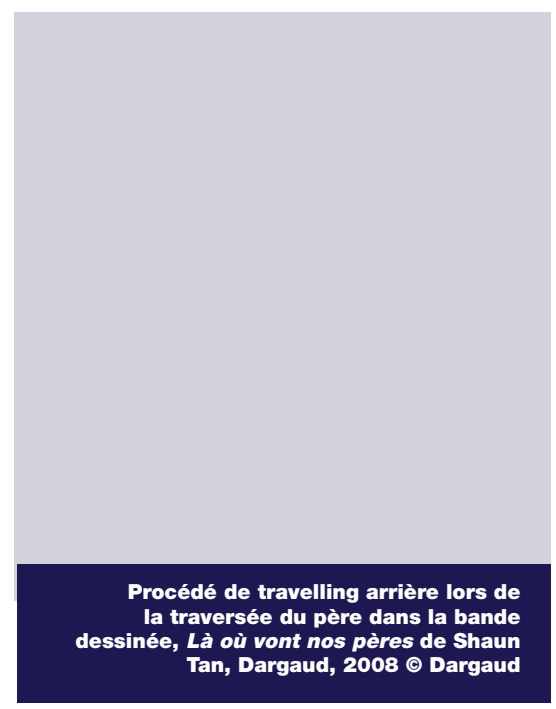
signification, puisque les gestes du père de famille sur le départ sont commencés dans l'image et se poursuivent entre les cases dans l'imaginaire des lecteurs. Penser le phénomène migratoire sous la plume de Shaun Tan permet d'explorer les manières passées, présentes et futures de penser le phénomène migratoire. En ce sens, l'approche esthétique de la migration s'articule dans Là où vont nos pères entre réinterprétation, décontextualisation mais aussi imagination et surréalisme. In fine, si l'objectif de Shaun Tan est de transcender ses sources premières pour produire un album égaré provenant d'un autre monde, on peut effectivement qualifier son ceuvre d'objet culturel non identifié.

\section{Bibliographie}

- Nancy Green, Repenser les migrations, Paris, PUF, 2002.

- Thierry Groensteen, La Bande dessinée : un objet culturel non identifié, Mouthiers-sur-Boëme, Éditions de l'an 2, 2006.

- Scot McCloud, L'Art invisible, Paris, Delcourt, 2007 pour la traduction française.

- Éric Maigret et Matteo Stefanelli (dir.), La Bande dessinée : une médiaculture, Paris, Armand Colin, 2012.

- Shaun Tan, Recherches sur un pays sans nom, l’art de là où vont nos pères, Paris, Dargaud, 2010.

- Shaun Tan, Là où vont nos pères, Paris, Dargaud, 2008. http://avrr.belgium.iom.int/en/other-projects/brazil-research.html 


\section{Notes}

1. Shaun Tan, Recherches sur un pays sans nom, l'art de là où vont nos pères, Paris, Dargaud, 2010.

2. Shaun Tan, Recherches sur un pays sans nom, l'art de là où vont nos pères, Paris, Dargaud, 2010.

3. L'expression est empruntée à Thierry Groensteen dans son ouvrage : La Bande dessinée : un objet culturel non identifié, Mouthiers-sur-Boëme, Éditions de l'an 2, 2006.

4. Shaun Tan, Recherches sur un pays sans nom, op. cit.

5. Ibid.

6. Voir à ce sujet, L'Arbre rouge chez Gallimard jeunesse, 2010, pour la traduction française ou encore Contes de la banlieue lointaine.

7. Il s'agit du titre original de la bande dessinée Là où vont nos pères, Paris, Dargaud, 2008.

8. Shaun Tan, Recherches sur un pays sans nom, op. cit.

9. L'expression est empruntée à Pierre Nora dans ses Lieux de Mémoires, Paris, Gallimard, 1984.

10. Shaun Tan, Recherches sur un pays sans nom, op. cit.

11. Le mot "religion" vient du latin relegere signifiant "relire" et religare signifiant "relier".

12. Nancy Green, Repenser les migrations, Paris, PUF, 2002.

13. Shaun Tan, Recherches sur un pays sans nom, op. cit., p. 5 .

14. Selon Nancy Green dans son ouvrage Repenser les migrations, op. cit., : "Le langage dans lequel les éléments liquides traversent des surfaces poreuses a été mis en place dès la fin du XIX' siècle."

15. L'expression est empruntée à Scot McCloud dans L'Art invisible, Paris, Delcourt, 2007 pour la traduction française. 